\title{
A COMPARATIVE STUDY OF THERESTORATIVE EFFECTS OF OCIMUM GRATISSIMUM AND OCIMUM CANUM ON ALCOHOL INDUCED HEPATOTOXICITY IN ALBINO RATS
}

\section{George $S^{1}$, Chaturvedi $P^{2 *}$ and B Moseki ${ }^{2}$}

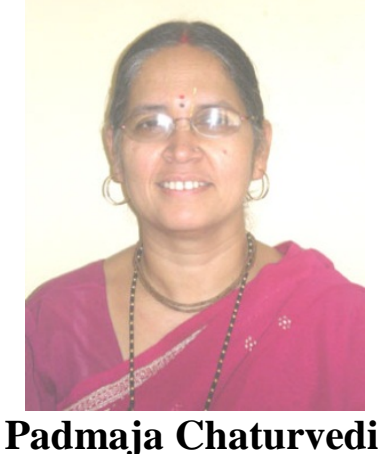

*Corresponding author email: chaturve@mopipi.ub.bw

${ }^{1}$ Department of Basic Sciences, Botswana College of Agriculture, University of Botswana, Gaborone, Botswana.

${ }^{2}$ Department of Biological Sciences, Faculty of Science, University of Botswana, Gaborone, Botswana. Pvt Bag 0022. 


\section{ABSTRACT}

Many species of Ocimum possess anti-inflammatory, antioxidant and anti-microbial properties and the plants are widely used in Ayurveda. The oxidative stress induced by ethanol consumption had been implicated the changes in the structure and functions of liver cells especially the membrane phospholipids leading to lipid peroxidation. This study was to assess the curative potential of the methanol extract of two different species of Ocimum, O. canum and O. gratissimum on alcohol induced hepatotoxicity in albino rats. Male Wister rats of body weight 200-250 g, were divided into 6 groups of six rats each as (1) NC - normal control with normal diet (2) EC - alcohol control fed on alcohol for thirty days; (3) EX -1 MEOG (methanol extract of Ocimum gratissimum) only to check the toxicity of the extract, (4) EX - 2 MEOC (methanol extract of Ocimum canum) only to check whether the extract had any toxic effect of its own and (5) EX - 3 MEOG after treating with ethanol for thirty days and confirming the hepatotoxicity, and (6) EX - 4 MEOC after the ethanol treatment. The rats were sacrificed after 30 days, and the blood collected for biochemical estimations. The results indicated that there were significant difference between the experimental rats (EX-groups) when compared with alcohol control (ECgroup) in all the parameters checked and they were not significantly different from the normal control (NC-group). The curative effect was established in groups 5 and 6, which showed a significant difference in antioxidant levels of all the parameters checked and retrieved its antioxidant status almost to the normal control. To come to the absolute normal condition of antioxidant levels it might have been needed a prolonged treatment for a longer period. Again, the groups, treated with extracts only, did not show any negative effect, which clearly indicated that the extract had no toxic effects. The use of antioxidant is an important preventive and curative method to minimize the pathological and toxic effects of oxidative stress induced by ethanol toxicity. Thus from the result obtained we conclude that MEOG and MEOC could effectively restore the antioxidant system from the oxidative stress induced by ethanol toxicity. In addition, there were no significant differences between the curative effects of these two species of Ocimum in terms of their effect on oxidative stress induced by ethanol toxicity.

Key words: Ocimum gratissimum, Ocimum canum, Alcohol, Hepatotoxicity, Albino rats 


\section{INTRODUCTION}

Oxidative stress plays an important role in the development of alcohol induced tissue injury. It is generally considered as an imbalance between peroxidant / antioxidant systems [1]. The major destructive effect is the initiation of lipid peroxidation leading to the destruction of cellular membrane causing leakage of lysomal enzyme and subsequent autolysis [2]. Chronic alcohol consumption is directly related to the induction of liver damage and the toxic effects of ethanol are attributed to increased generation of free radicals and the development of state of oxidative stress.

Increased oxidative stress occurs directly due to ethanol and its oxidative products. Ethanol is reported to be converted to ethyl and 1- hydroxyethyl radicals [3]. Recent findings indicate that free radicals species, namely hydroxyethyl radicals are generated during ethanol metabolism by microsomal monooxygenase system and particularly by the ethanol -inducible form of cytochrome P-450 (CYP2E1) that enhances liver damage by chronic ethanol exposure [4, 5]. Several mechanisms including, the depletion of antioxidants, such as vitamin $\mathrm{E}$ and glutathione might account for the oxidative stress associated with ethanol intoxication [6]. Despite of decades of research, the role of free radicals in alcohol-induced organ injury is still a matter of debate. For a long period plants have been a valuable source of natural product for maintaining human health, especially in the last decade, more intensified studies on natural therapies established this.

The use of plant extracts and phytochemicals derived from medicinal plants are extensively used for pharmaceutical purposes. Therefore, such plants should be investigated to understand better their properties, safety and efficiency. The antioxidant activity or the inhibition of the generation of free radicals is important in providing protection against hepatic damage and a number of plants possess the potential hepatoprotective properties by improving antioxidant status. Many synthetic antioxidant components have shown toxic and/or mutagenic effects, which have shifted the attention towards the naturally occurring antioxidants [8].

Indian medicinal plants belonging to about 40 families were investigated for liver protective or curative functions [7]. One of the above-mentioned families is Lamiaceae, which includes the basil family. Ocimum species are known for their medicinal properties [8] and are used extensively in Ayurvedic preparations. $O$. canum belongs to Lamiaceae and it is a branched shrub of about 0.5 to 1 meter height, with round stem and simple, hairy leaves, which are oppositely arranged with purple inflorescence. O. gratissimum belongs to the same genus commonly known as wild basil also belongs to Lamiaceae family, highly branched shrub of about 1-2 meters height, with ribbed stem and laborious branches. Its leaves are simple, oppositely arranged, 3-5 centimeters long, 1.2 centimeters wide. $O$. canum possess antibacterial, mosquito repellent properties and hepatoprotective property [9, 10, and 11]. It lowers the blood glucose level and facilitates insulin released by isolated pancreatic beta islets cells [12]. O. gratissimum is used for nasal congestion cough and abdominal pain in Africa. It is reported to have antidiabetic and antioxidant properties [13, 14]. 
The present study explored more on the restorative effect of two different species of Ocimum (O. canum and O. gratissimum) and evaluated the curative effect of these extracts on alcohol-induced hepatotoxicity in albino rats. The study also compared the effect of both extracts in oxidative stress induced by ethanol toxicity. Presence of any toxic effect or side effect when these plants were administered as supplementation was also checked.

\section{MATERIALS AND METHODS}

\section{Plant Material}

Plant extract: Tender part of $O$. canum and $O$. gratissimum that is the tip of the branches were collected locally from Botswana around Gaborone and identified to the nearest taxonomy level. The voucher specimen was submitted in the herbarium and voucher No. for Ocimum canum was 2006/G, A01 and for Ocimum gratissimum was 2006/G, A02.

\section{Preparation of Extract}

The plant was cut into small pieces and dried in the shade, then coarsely powdered and soaked in $70 \%$ methanol for three days at room temperature. The extract was filtered make it solvent free by using Buchi type rotary evaporator $\left(65^{\circ} \mathrm{C}\right)$ and the yield were7.8 \% and $7.2 \%(\mathrm{~W} / \mathrm{W})$ respectively. The extract was administered to rats orally after dissolving it in the drug carrier (distilled water + tween-80 in the ratio of 9:1) with rubber tubing attached to a syringe.

\section{Animals}

Male albino rats, Wister strain of body weight ranging 200-250 g were housed in colony cages at an ambient temperature of $25^{\circ} \mathrm{C} \pm 2{ }^{\circ} \mathrm{C}$ and $50-55 \%$ relative humidity with 12 hours light dark cycle. They had water and food ad libitum .The experiment was conducted as per internationally accepted principles for laboratory animal care unit of University of Botswana (CST 16/9 III25)

\section{Chemicals}

All the chemicals used were analytical grade and bought from Sigma-Aldrich Chemical Company, (St. Louis, MO) USA.

\section{Experimental Design}

This experiment was designed to find out the curative effect of the extract on alcoholinduced hepatotoxicity. Animals were divided into 6 following groups and all groups had six rats each. Group 1 of six was kept as normal control on normal diet and distilled water. Group 2 was given the ethanol for 30 days initially and later with normal diet as alcohol control. Group 3 and 4 were treated with extracts only to check the toxic effect. Group 5 and 6 were treated with alcohol for 30 days to induce hepatotoxicity prior to the administration of the extracts and given MEOC and MEOG, respectively for thirty days. 
Group 1- Normal control-fed on normal diet and distilled water.

Group 2- Alcohol control (5g/kg body wt/day) for 30-days and later on normal diet and distilled water for 30 days.

Group 3- MEOC only (100 mg/kg body wt/day) with normal diet and distilled water for 30 days.

Group 4- MEOG only (100 mg/kg body wt/day) with normal diet and distilled water

Group 5- MEOC after alcohol (100 mg/kg body wt/day) with normal diet and distilled water for 30 days.

Group 6- MEOG after alcohol (100 mg/kg body wt/day) with normal diet and distilled water for 30 days.

Treatment was carried out for 30 days and at the end of the experimental period the rats were sacrificed to collect the blood for biochemical estimations.

\section{Biochemical Analysis}

\section{Estimation of Thiobarbituric Acid Reactive Substances}

TBARS in plasma was estimated by the method described by method of Niehaus and Samuelsson with little modifications [15]. Lipid peroxidation was measured by the formation of thiobarbituric acid reactive substances (TBARS) such as malondialdehyde (MDA). MDA has been identified as the product of lipid peroxidation that reacts with thiobarbituric acid to give red species absorbing at 535 nm. $0.1 \mathrm{ml}$ of plasma was treated with $2 \mathrm{ml}$ of TCA-TBA-HCL $(1: 1: 1)$ mixture and incubated in boiling water bath for ten minutes. The mixture was cooled and was mixed with $2 \mathrm{ml}$ of freshly prepared $1 \mathrm{~N} \mathrm{NaOH}$. Absorbance was measured at $535 \mathrm{~nm}$.

\section{Estimation of Reduced Glutathione}

Reduced glutathione was estimated by the method of Ellman [16]. $0.25 \mathrm{ml}$ of plasma was mixed with $0.5 \mathrm{ml}$ of precipitating buffer (5\%TCA in $1 \mathrm{~m} \mathrm{M}$ EDTA) and centrifuged. The supernatant was collected and mixed with $2.5 \mathrm{ml}$ of $0.1 \mathrm{M}$ phosphate buffer (pH 8.0). Colour was developed by adding $100 \mu \mathrm{l}$ of DTNB (0.01\%) and the absorbance was recorded at $412 \mathrm{~nm}$.

\section{Estimation of Super-oxide dismutase}

Super-oxide dismutase was assayed by the method of Kakkar [17]. The assay of SOD activity was based on the principle of inhibitory effects of SOD on reduction of nitroblue tetrazolium dye. A single unit of enzyme was expressed as $50 \%$ inhibition of NBT (Nitroblue tetrazolium) reduction $/ \mathrm{min} / \mathrm{mg}$ protein. The reaction mixture contained $150 \mu \mathrm{l}$ EDTA, $600 \mu \mathrm{l}$ L-methionine, $300 \mu \mathrm{l}$ NBT and make the volume up to $2.8 \mathrm{ml}$ by the addition of SOD buffer. To the reaction mixture, was added $200 \mu \mathrm{l}$ of the lysate except in the control. Finally to this was added $200 \mu \mathrm{l}$ of riboflavin to start the reaction. The test tube was kept under a fluorescent lamp and the reaction kinetics was measured up to four minutes at 560nm. 


\section{Estimation of Catalase}

Catalase was estimated by the method of Hans Bisswagner [18]. To $0.98 \mathrm{ml}$ of $\mathrm{H}_{2}$ $\mathrm{O}_{2}$ solution $(10 \mathrm{mM}), 0.2 \mathrm{ml}$ of plasma was added .Absorption was recorded at 240 $\mathrm{nm}$ and the catalase activity was calculated using the extinction coefficient of $\mathrm{H}_{2}$ $\mathrm{O}_{2}$ 0.071). The activity was expressed as micromoles of $\mathrm{H}_{2} \mathrm{O}_{2}$ oxidized per minute per milligram protein.

\section{Estimation of Vitamin E ( $\propto$ Tocopherol)}

$\propto$ - Tocopherol was estimated by the method of Martinek [19]' Tocopherol was precipitated in the xylene layer by centrifuging the mixture Tocopherols reduce the Fe (111) ions to Fe (11) ions. These ions formed a red complex with $\propto-\propto-$ Dipyridyl reagent and it was measured as the color range at $520 \mathrm{~nm}$. About $0.5 \mathrm{ml}$ of serum was taken in a centrifuge tube and added $0.5 \mathrm{ml}$ of xylene to it Similarly $0.5 \mathrm{ml}$ of distilled was taken for the blank and $0.5 \mathrm{ml}$ of standard solution for the standard. $0.5 \mathrm{ml}$ of xylene was added to all these centrifuge tubes. Close the tubes tightly, mix them thoroughly and centrifuged. The xylene layer (containing the precipitated tocopherol) was then carefully pipetted into clean tubes and mixed with $0.35 \mathrm{ml} \propto-\propto$ ' Dipyridyl reagent. The absorbance of test samples was measured against a blank at 460nm.

\section{Estimation of Vitamin-C (Ascorbic Acid)}

Ascorbic acid was assayed in plasma by the method of Roe with slight modification [20]. To $0.5 \mathrm{ml}$ plasma, $1.5 \mathrm{ml}$ of $4 \%$ TCA was added and protein was precipitated by centrifuging at 3000 rpm for 20 minutes. The supernatant was used to estimate the ascorbic acid content. One ml of supernatant was mixed with $0.5 \mathrm{ml}$ of DNPH reagent (2g dinitrophynyl hydrazine, $250 \mathrm{~g}$ thiourea, $30 \mathrm{mg} \mathrm{CuSO}_{4} \cdot 5 \mathrm{H}_{2} \mathrm{O}$ in $100 \mathrm{ml} 9 \mathrm{~N}$ sulphuric acid) and was incubated for 3 hours at $37{ }^{\circ} \mathrm{C} .2 .5 \mathrm{ml}$ of $85 \%$ sulphuric acid was added to develop the color. The absorbance was recorded at $540 \mathrm{~nm}$ after 30 minutes.

The kits bought from Sigma estimated were used to estimate AST and ALT and the manufacture's guidelines were strictly followed.

\section{Statistical Analysis}

All data are expressed as the mean \pm S.E. mean of $n=6$. Analysis of variance was performed by one-way ANOVA and the significant differences between the means were determined by Holm-Sidak method at $p$-value of $\leq 0.05$. Statistical software Sigmastat 3.1 was used to analyze the data.

\section{RESULTS}

To evaluate the restorative effect of the extract on alcohol induced hepatotoxicity groups 5 and 6 were fed with ethanol for 30 days to induce the liver toxicity and conducted biochemical investigations to establish the same then compared with the ethanol control group. After the confirmation, these groups were given the extracts for 30 days (100 mg/kg body wt/day) and the biochemical assays were conducted then 


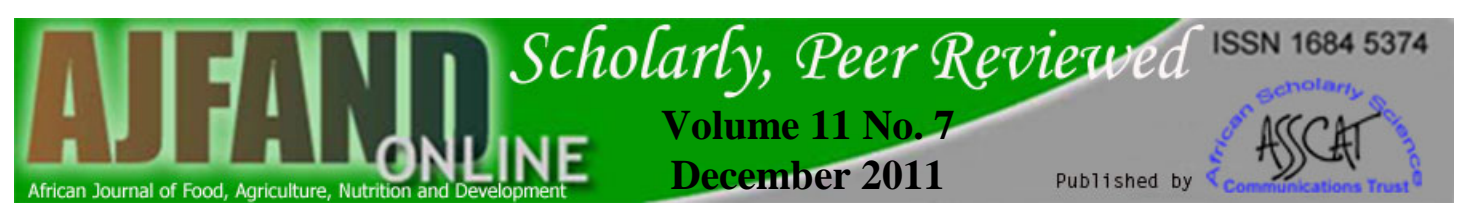

again compared with the NC and EC groups. Simultaneously another two groups 3 and 4 were given the extract only for 30 days to check whether the extract has any toxic or side effects.

Alcohol administration increased liver function biomarkers ALT and AST significantly in comparison with normal control (Table 1) but the treatment with the extract for 30 days helped them to come back almost to the normal condition. Same way the lipid peroxidation was indicated by TBARS, which was elevated to four times more when compared to the normal control. The results of the lipid peroxidative indices revealed the fact that during ethanol administration the free radicals were produced by ethanol metabolism, which caused the hepatocytes to undergo the oxidative stress. The elevation of the TBARS as well as the levels of the liver markers such as ALT and AST clearly indicated that the lipid membrane structure was damaged causing the leakage of these enzymes to the blood. The Administration of MEOC and MEOG to alcohol-treated rats reduced lipid peroxidation levels to those measured in the control rats. Statistical analysis of these data indicates that extracts significantly reduced $(P<0.05)$ LPO levels in groups treated with the extracts.

For studying the effect of enzymatic antioxidant status, the activities of enzymatic antioxidants such as SOD, CAT (Table 2) were considered. The activities of CAT and SOD significantly increased in groups 5 and 6 when compared with EC group and almost reached to the same level as the normal control. $(P<0.001)$. Table-3 is the curative effect of MEOC and MEOG after treating with ethanol. Normal control is significantly different from the alcohol treated groups $(P<0.001)$ but not showing the significant difference from both the experimental groups in all the parameters checked. The experimental groups were showing slight difference from the normal control but they are not significantly different. All the above results show the normal control group is significantly different from the EC group but did not show any significant difference with any experimental groups.

\section{DISCUSSION}

Alcohol-induced liver injury can be linked to oxidative stress and increased production of reactive oxygen species [1]. Many pathways have been suggested to contribute to the ability of ethanol to induce a state of oxidative stress, which involve increases of lipid peroxidation and protein oxidative and nitrosative stress and a decrease in hepatic antioxidant defense [2,3]. The mechanisms by which alcohol causes liver injury are still not clear.

Free radicals have aroused significant interest among scientists in the past decade and looking at different aspects of the causative agents of this damage. The increase in plasma liver markers is a direct reflection of oxidative injury of the liver tissue. Lipid peroxidation, as reflected in TBARS values were higher in all ethanol treated groups when compared to normal controls and experimental groups clearly indicate the oxidative stress in hepatic and extra hepatic tissues due to ethanol and its oxidation. (Table1) Various pathways play a role in ethanol induced tissue injury, including changes in cellular oxidized NAD+, NADH and production of acetaldehyde protein

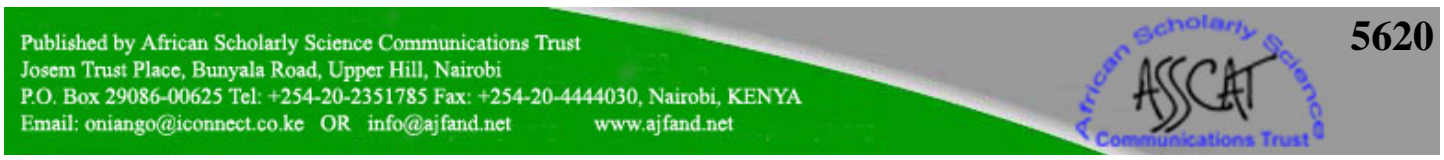


adducts [21]. It also produces induction of CYP2E1 formation of 1-hydroxyethyl free radicals ethanol mediated mitochondrial damage [22]. These changes cause lipid peroxidation in cellular membranes in hepatic tissues and cause severe damage and leakage of liver markers into the circulation.

ALT and AST are transaminases localized in the hepatic cells are released in the circulation after hepatic cell damage [23]. High levels of ALT and AST in EC groups indicates hepatic cell damage by ethanol byproducts that caused lipid peroxidation (Table1). Levels of these enzymes were also low in EX1 and EX2, where the rats were administered extracts only and this shows that these extracts do not have any toxic effects. Low levels of these enzymes in experimental groups EX3 and EX4 indicate restoration of the normal condition of hepatic cells to almost to the normal control.

Enzymatic antioxidants such as SOD and CAT showed significant difference in AC groups when compared with EX groups and NC groups clearly indicates the extent of toxicity and depletion of these enzymes. (Table2) CAT helps in neutralizing the toxic effect of $\mathrm{H}_{2} \mathrm{O}_{2}$ and converts it to water and non reactive oxygen species thus prevents the generation of hydroxyl radicals and protects the cells from oxidative damage. This could be supported by glutathione peroxidase and they constitute a mutually supportive team of defence against ROS [24]. In this experiment, also treating with the extracts after alcohol treatment could restore the depleted CAT and SOD activities almost to the normal condition Super oxide dismutase catalyzes the dismutation of super oxide into oxygen and hydrogen peroxide [25]. As such, it is an important antioxidant defence in nearly all cells exposed to oxygen. Our results support this finding. In this experiment, degree of catalase and SOD activities is very low in EC group as compared to EX groups where their levels do not differ significantly from the normal control group NC. These findings again support the participation of these two plants in maintaining the normal antioxidant system. Values in EX2 and EX3 do not differ significantly from NC and this again supports the view that these plants do not have any depleting effects on this enzymatic antioxidant system.

Glutathione is a tripeptide present in all mammalian cells; it participates in many metabolic processes for the protection of cells against free radicals and toxic metabolites. The decrease in GSH levels represents the increased utilization due to oxidative stress [26]. This serves as a substrate for the synthesis of glutathione-related enzymes; GSH acts as a free radical scavenger, a regenerator of tocopherol, and plays an important role in the maintenance of protein sulfhydryl groups.

The levels of vitamin E and C were also decreased significantly in EC groups when compared with the NC and all experimental groups. This is in agreement with the previous reports that chronic alcoholics are deficient in vitamin $C$ and $E$ [27]. The increased oxidative stress due to alcohol ingestion might have resulted in complete utilization of vitamin $\mathrm{C}$ and $\mathrm{E}$ thus depleting their levels. There was no significant difference between the NC groups and all experimental groups for O. gratissimum and $O$. canum. Hence it appears to be very clear that the extracts were involved in quenching of free radicals by participating in detoxification reactions through GSH, 
Vitamin C and Vitamin E. These results also support the fact that these two plants are participating well in boosting the antioxidant system.

The use of antioxidants was recognized as one of the important counter measures against the toxic effects of any type oxidative stress. Comparison with of these results with other species of Ocimum also shows almost the same type of effects [28]. Again, when comparing the results of the effects of these two species of Ocimum, O. gratissimum and $O$. canum in all the above parameters checked showed almost the same type of results explains that they work in the same pathway in the antioxidant system. Our results suggest that these extract possess potent antioxidative activity and protects liver against ethanol induced oxidative stress probably via the alteration of cytochrome P-450. It was reported that these two species indigenous to Africa has potential antioxidant properties to prevent the oxidative stress induced by ethanol intoxication [29].

In conclusion, the methanol extracts of these two species $O$. gratissimum and $O$. canum protects liver tissue against oxidative damage and could be used as an effective protector against ethanol induced hepatic damages. Further works are needed to fully characterize the responsible active principle(s) present in the plant and elucidate its possible mode of action and that is in progress.

\section{ACKNOWLEDGEMENT}

The authors are thankful to Research and Publication Committee of University of Botswana, for providing funds to carry out this work. 


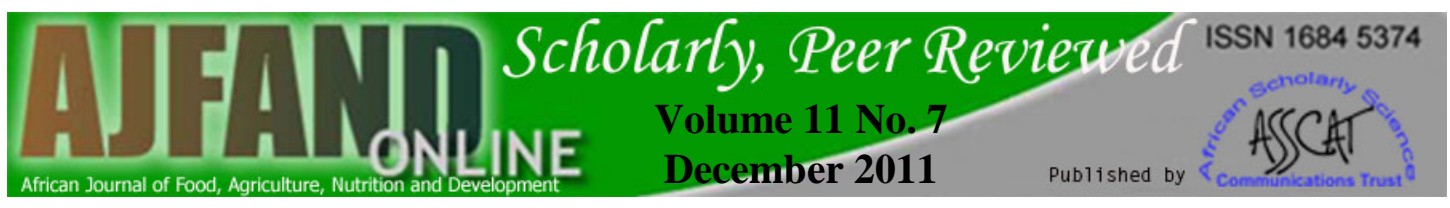

Table 1: Plasma levels of lipid peroxidative indices of ethanol induced oxidative stress

\begin{tabular}{lllll}
\hline S No. & Groups & \multicolumn{3}{c}{ Parameters } \\
& & $\begin{array}{l}\text { ALT } \\
\text { UBARS }\end{array}$ & AST \\
nmol/L & & & \\
\hline & & & & \\
1 & NC & $1.52 \pm 0.57$ & $71.03 \pm 0.78$ & $39.18 \pm 0.92$ \\
2 & EC & $6.17 \pm 0.83^{*}$ & $39.92 \pm 0.54^{*}$ & $63.61 \pm 0.67$ \\
3 & EX1 & $1.36 \pm 0.65$ & $38.93 \pm 0.09$ & $38.89 \pm 0.45$ \\
4 & EX2 & $1.33 \pm 0.69$ & $43.68 \pm 0.65$ & $38.78 \pm 0.56$ \\
5 & EX3 & $1.57 \pm 0.94$ & $42.66 \pm 0.23$ & $43.86 \pm 0.32$ \\
6 & EX4 & $1.54 \pm 0.53$ & & \\
\hline
\end{tabular}

$\mathrm{n}=6$ in each group, ${ }^{*}$ shows significant difference and the results were given as mean \pm SEM.P $\leq 0.05$

Group 1- NC: Normal control on normal diet

Group 2- AC: Alcohol control (5gms/kg body wt/day.

Group 3- EX1: Experimental rats administered with MEOG only. (100mg/Kg, body weight)

Group 4 -EX2: Experimental rats administered with MEOC only. (100mg/Kg body weight)

Group 5 - EX3: Experimental rats administered with MEOG after treated with ethanol. (100mg/Kg body weight)

Group 6 - EX4: Experimental rats administered MEOC after treated with ethanol. (100mg/Kg body weight.) 
Table 2: Levels of enzymatic antioxidants of Red Blood Cell of ethanol induced oxidative stress in albino rats.

S No. Groups $\quad$ Parameters

$\begin{array}{ll}\text { SOD } & \text { CATALASE } \\ (\mathrm{U} / \mathrm{mg} \text { of } \mathrm{Hb}) & \mathrm{U} / \mathrm{mg} \text { of } \mathrm{Hb})\end{array}$

\begin{tabular}{llll}
\hline 1 & NC & $2.54 \pm 0.13$ & $46.43 \pm 0.49$ \\
2 & EC & $1.40 \pm 0.18^{*}$ & $30.05 \pm 0.43$ \\
3 & EX1 & $2.53 \pm 0.06$ & $47.18 \pm 0.77$ \\
4 & EX2 & $2.68 \pm 0.13$ & $46.98 \pm 0.43$ \\
5 & EX3 & $2.36 \pm 0.05$ & $45.55 \pm 0.17$ \\
6 & EX4 & $2.29 \pm 0.89$ & $46.17 \pm 0.19$ \\
\hline
\end{tabular}

$\mathrm{n}=6$ in each group ${ }^{*}$ shows significant difference and the results were given as mean \pm SEM.P $\leq 0.05$

Group 1- NC: Normal control on normal diet

Group 2- AC: Alcohol control (5gms/kg body wt/day.

Group 3- EX1: Experimental rats administered with MEOG only. (100mg/Kg, body weight).

Group 4 -EX2: Experimental rats administered with MEOC only. (100mg/Kg body weight).

Group 5 - EX3: Experimental rats administered with MEOG after treated with ethanol. (100mg/Kg body weight)

Group 6 - EX4: Experimental rats administered MEOC after treated with ethanol. (100mg/Kg body weight.) 
Table 3: Plasma levels of nonenzymatic antioxidants of ethanol induced oxidative stress in albino rats

\begin{tabular}{lllll} 
S No. & Groups & \multicolumn{2}{c}{$\begin{array}{c}\text { Parameters } \\
\text { VIT-E } \\
\text { mg/dl }\end{array}$} & $\begin{array}{l}\text { GSH } \\
\text { mg/dl }\end{array}$ \\
\hline & & & & \\
1 & NC/dl & $2.57 \pm 1.10$ & $2.56 \pm 0.13$ & $34.20 \pm 0.52$ \\
2 & EC & $1.26 \pm 0.07$ & $1.04 \pm 0.04$ & $18.06 \pm 0.77$ \\
3 & EX1 & $2.64 \pm 0.19$ & $2.53 \pm 0.11$ & $36.78 \pm 0.31$ \\
4 & EX2 & $2.54 \pm 0.36$ & $2.58 \pm 0.62$ & $35.88 \pm 0.31$ \\
5 & EX3 & $2.23 \pm 0.14$ & $2.42 \pm 0.08$ & $32.66 \pm 0.38$ \\
6 & EX4 & $2.33 \pm 1.00$ & 2.390 .12 & $32.30 \pm 0.22$ \\
\hline
\end{tabular}

$\mathrm{n}=6$ in each group ${ }^{*}$ shows significant difference and the results were given as mean \pm SEM.P $\leq 0.05$

Group 1- NC: Normal control on normal diet

Group 2- AC: Alcohol control (5gms/kg body wt/day.

Group 3- EX1: Experimental rats administered with MEOG only. (100mg/Kg, body weight).

Group 4 -EX2: Experimental rats administered with MEOC only. (100mg/Kg body weight).

Group 5 - EX3: Experimental rats administered with MEOG after treated with ethanol.

(100mg/Kg body weight)

Group 6 - EX4: Experimental rats administered MEOC after treated with ethanol. (100mg/Kg body weight) 


\section{REFERENCES}

1. Nordmann $\mathbf{R}$, Ribière $\mathbf{C}$ and $\mathbf{H}$ Rouach. Implication of free radical mechanisms in ethanol-induced cellular injury. Free Radic. Biol. and Med. 1992; 12: 219-240.

2. Finkel T and NJ Holbrook Oxidants, oxidative stress and the biology of ageing. Nature 2000; 408: 831- 49

3. Reinke LA, Moore DR, and Hague CM and PB McCay Metabolism of ethanol to 1-hydroxyethyl radicals in rat liver microsomes: comparative studies with three spin trapping agents. Free. Radic. Res. 1994; 24 (4):213-22.

4. Kono H, Rusyn I, Yin M and SG Yamashina NADPH oxidase-derived free radicals are key oxidants in alcohol-induced liver disease. J. Clinic. Invest. 2000; 106: 867-872

5. Lieber CS Cytochrome P-4502E1: its physiological and pathological role. Physiol Rev 199; 77: 517-544

6. Lieber CS Hepatic, metabolic and nutritional disorders of alcoholism: from pathogenic to therapy. Review: Crit. Rev. Clin. Lab. Sci. 2000; 37(6): 551-84.

7. Geetha RK and DM Vasudavan Inhibition lipid peroxidation by botanical extracts of Ocimum sanctum in vivo and in vitro studies. Life Sci. 2004; 19: $21-28$

8. Handa SS, Sharma A and KK Chakaraborti Natural products and plants as liver protecting drugs. Fitoter, 1986; 57: 307-45

9. Janssen AM, Scheffer JJ, Nterzurubanza $L$ and AS Baerheimthe Antimicrobial activities of some Ocimum species grown in Rwanda. Journal of Ethnopharmacol..1989; 26(1): 57-63.

10. Palsson K and TG Jaenson Plant products used as mosquito repellents in Guinea Bissau, West Africa. Acta Tropica; 1999; 2 (1): 39-52.

11. Nyarko AK, Asare Anase H, Ofosuhene $M$ and ME Addy Extract of Ocimum canum lowers blood glucose and facilitates insulin release by isolated pancreatic beta-islet cells Phytomedicine 2002; 5( 9) :346-51.

12. George S, Chaturvedi P, Raseroka BH, Mpho $M$ and $\mathbf{N}$ Makate Hypoglycemic effect of methanol extract of Ocimum suave on albino rats. Journal of Appl. Zool. Res. 2004; 15 (2): 189-194.

13. Chaturvedi P, George S and A John Preventive and protective effects of wild Basil in ethanol -induced liver toxicity in rats. Br. J. of Biomed. Sci. 2007; 64(1): 10-12. 
14. George $\mathbf{S}$ and $\mathbf{P}$ Chaturvedi Protective role of Ocimum canum plant extract in alcohol-induced oxidative stress in albino rats. Br. J. of Biomed. Sci.2008; 65(2): 1-6.

15. Niehaus WG Samuelson B. Formation of MDA from phospholipids arachidonate during microsomal lipid peroxidation. Euro J. Biochem 1986; 6: 126- 130

16. Ellman GL Tissue sulhydryl groups Arch. Biochem. Biophys 1959; 82:70-77

17. Kakkar P, Das B and PN Viswanathan A modified spectrophotometric assay of superoxide dismutase .Indian Journal of Biochemistry and Biophysis 1984; 21:130-132.

18. Bisswanger H Practical enzymology Wiley-VCH 2004: 79

19. Martinekn RG Method for the determination of Vitamin E (total tocopherols) in serum. Clinical Chemistry 1964: 10: 1078-86.

20. Roe JH Methods of biochemical analysis. New York Interscience 1961.

21. Pari $\mathbf{L}$ and $\mathbf{M}$ Latha Protective role of Scoparia dulcis extract on brain antioxidant status and lipid peroxidation in STZ diabetic male Wistar rats. BMC Compliment. Alternat. Med. 2004; 2.doi :(10) 1186/ 1472-6882-4-16

22. Bandhopadhy U, Das $\mathbf{D}$ and KR Banerjee Reactive oxygen species: Oxidative damage and pathogenesis. Current Science. 1999; 77: 658-665.

23. Jaya DS, Augustine $\mathbf{J}$ and VP Menon Role of lipid peroxides, glutathione and antiperoxidative enzymes in alcohol and drug toxicity. Ind. J. Exp. Biol. 1993; 31:453-459.

24. Husain $\mathbf{K}$ and SM Somani Interaction of exercise and ethanol on hepatic and plasma antioxidant system in rat. Pathophysiol. 1997; 4:69-74.

25. Speisky H, MacDonald A, Griles G, Orrego $\mathbf{H}$ and $\mathbf{L}$ Israel Increased loss and decreased synthesis of hepatic glutathione after acute ethanol administration. Biochem J 1985; 255: 565-572.

26. Guerri $\mathbf{C}$ and S Grisolia Changes in glutathione in acute and chronic alcohol intoxication. Pharmacol. Biochem. Behavior1980; 13: 53-61.

27. Sivaram AG, Suresh MV and M Indra Combined effect of ascorbic acid and selenium supplementation on alcohol-induced oxidative stress in guinea pigs. Comp. Biochem. Physiol. Toxicol. Pharmacol. 2003; 134(3): 397-401.

28. Karthikayan K, Gunasekaran P, Ramamurthy $\mathbf{N}$ and S Govindasamy Anti cancer activity of Ocimum sanctum. Pharmaceutical Biol 1999; 37: 228290. 


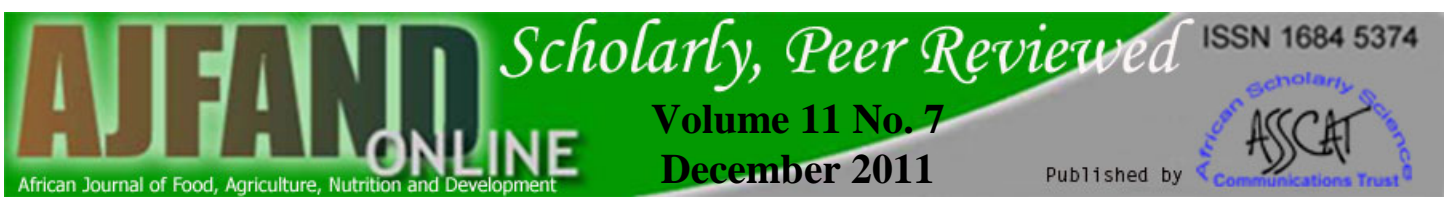

29. George $\mathbf{S}$ and $\mathbf{P}$ Chaturvedi A comparative study of the antioxidant properties of two different species of Ocimum of Southern Africa on alcohol induced oxidative stress. J. Med. Food.2009 12 (5): 1154-58. 